\title{
RESULTANTS OF MATRIX POLYNOMIALS
}

\author{
BY I. C. GOHBERG AND L. E. LERER
}

Communicated by Chandler Davis, February 24, 1976

The $(n+m) \times(n+m)$ matrix

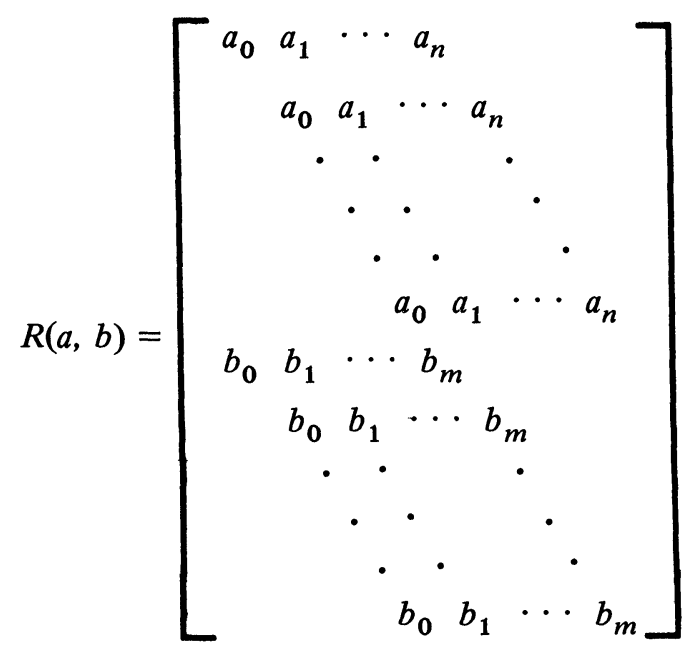

is called the resultant matrix of the two polynomials $a(\lambda)=a_{0}+a_{1} \lambda+\cdots+$ $a_{n} \lambda^{n}$ and $b(\lambda)=b_{0}+b_{1} \lambda+\cdots+b_{m} \lambda^{m}\left(a_{j}, b_{j}, \in \mathbf{C}^{1}, a_{n} \neq 0, b_{m} \neq 0\right)$. The determinant of this matrix is called the resultant of the polynomials $a(\lambda)$ and $b(\lambda)$. The following classical theorem on resultants is well known: The number of common roots (counting multiplicities) of the polynomials $a(\lambda)$ and $b(\lambda)$ is equal to $\operatorname{dim} \operatorname{Ker} R(a, b)$.

This statement does not admit a straightforward generalization to matrix polynomials [1], if the same definition of the resultant matrix $R(a, b)$ is used as in the one-dimensional case. For example the matrix

$$
R\left(\left(\begin{array}{cc}
\lambda-1 & 0 \\
1 & \lambda-1
\end{array}\right),\left(\begin{array}{cc}
\lambda & 1 \\
0 & \lambda-2
\end{array}\right)\right)
$$

is not invertible although the polynomial matrices do not have common eigenvalues, and the matrix

$$
R\left(\left(\begin{array}{cc}
\lambda+1 & 0 \\
1 & \lambda
\end{array}\right),\left(\begin{array}{ll}
\lambda & -1 \\
0 & \lambda+1
\end{array}\right)\right)
$$

AMS (MOS) subject classifications (1970). Primary 15A54; Secondary 15A24.

Key words and phrases. Linear algebra, resultant, matrix polynomial, resultant matrix, $\lambda$-matrix, common eigenvalue, multiplicity of a common eigenvalue. 
is invertible although the eigenvalues of the polynomials coincide.

The main result of this article is concerned with a new definition of a resultant matrix $R^{\otimes}(a, b) \stackrel{\text { def }}{=} R(a \otimes I, I \otimes b)$, where $\otimes$ is the sign of the righthand tensor product.

Let $\lambda_{1}, \lambda_{2}, \ldots, \lambda_{r}$ be all the common eigenvalues of $a(\lambda)$ and $b(\lambda)$. Let us denote by $k_{1 p}(a) \geqslant k_{2 p}(a) \geqslant \cdots \geqslant k_{j_{p} p}(a)$ the powers of the elementary divisors of $a(\lambda)$ for the eigenvalue $\lambda_{p}$. Let

$$
\mu\left(a, b, \lambda_{p}\right)=\sum_{l=1}^{j_{p}(a)} \sum_{j=1}^{j_{p}(b)} \min \left\{k_{l p}(a), k_{j p}(b)\right\}
$$

and

$$
\mu(a, b)=\left(\mu\left(a, b, \lambda_{1}\right)+\mu\left(a, b, \lambda_{2}\right)+\cdots+\mu\left(a, b, \lambda_{r}\right)\right) .
$$

The main result is the following generalization of the classical resultant theorem.

THEOREM. Let $a(\lambda)$ and $b(\lambda)$ be $d \times d$ matrix polynomials with invertible highest coefficients. Then $\operatorname{dim} \operatorname{Ker} R^{\otimes}(a, b)=\mu(a, b)$. Particularly $a(\lambda)$ and $b(\lambda)$ have a common eigenvalue if and only if $\operatorname{det} R^{\otimes}(a, b)=0$.

We start sketching the proof by defining the common multiplicity of the eigenvalue $\lambda_{0}$ of polynomials $a(\lambda)$ and $b(\lambda)$. Let $\phi_{0}, \phi_{1}, \ldots, \phi_{r}$ be a chain of the eigenvector $\phi_{0}$ and the associated vectors $\phi_{1}, \ldots, \phi_{r}$, which correspond to $\lambda_{0}$ :

$$
\sum_{j=1}^{k} \frac{1}{j !}\left(\frac{d^{j}}{d \lambda^{j}} a\right)\left(\lambda_{0}\right) \phi_{k-j}=\sum_{j=1}^{k} \frac{1}{j !}\left(\frac{d^{j}}{d \lambda^{j}} b\right)\left(\lambda_{0}\right) \phi_{k-j}=0 \quad(k=0,1, \ldots, r)
$$

The number $r+1$ is called the length of the chain. We denote the maximal length of such chain with the fixed vector $\phi_{0}$ by rank $\left(\lambda_{0}, \phi_{0}\right)$. It is easy to find a basis $\phi_{10}, \phi_{20}, \ldots, \phi_{j_{0}, 0}$ in the subspace $M=\operatorname{Ker} a\left(\lambda_{0}\right) \cap \operatorname{Ker} b\left(\lambda_{0}\right)$ such that $\operatorname{rank}\left(\lambda_{0}, \phi_{10}\right)=\max \operatorname{rank}\left(\lambda_{0}, \phi\right)(\phi \in M)$ and $\operatorname{rank}\left(\lambda_{0}, \phi_{j_{0}}\right)$ $=\max \operatorname{rank}\left(\lambda_{0}, \phi\right)\left(\phi \in M_{j} ; j=2,3, \ldots, r\right)$ where $M_{j}$ is the subspace with the basis $\phi_{j+1,0}, \phi_{j+2,0}, \ldots, \phi_{r, 0}$. It is easy to see that for every vector $\phi \in M$, the integer $\operatorname{rank}\left(\lambda_{0}, \phi\right)$ is equal to one of the numbers $k_{j}=\operatorname{rank}\left(\lambda_{0}, \phi_{j_{0}}\right)(j=$ $\left.1,2, \ldots, j_{0}\right)$. Therefore these numbers depend only on the polynomials $a(\lambda)$, $b(\lambda)$ and the eigenvalue $\lambda_{0}$. The integer $\nu\left(a, b, \lambda_{0}\right)=k_{1}+k_{2}+\cdots+k_{j_{0}}$ is called the common multiplicity of the eigenvalue $\lambda_{0}$ of the polynomials $a(\lambda)$ and $b(\lambda)$. If $M=\{0\}$, then we set $\nu\left(a, b, \lambda_{0}\right)=0$.

The proof of the theorem involves two main steps. The first is to prove the equality $\mu\left(a, b, \lambda_{0}\right)=\nu\left(a \otimes I, I \otimes b, \lambda_{0}\right)$. The main theorem from [1] then implies that for large $l$, $\operatorname{dim} \operatorname{Ker} R_{l}(a \otimes I, I \otimes b)=\mu(a, b)$, where 


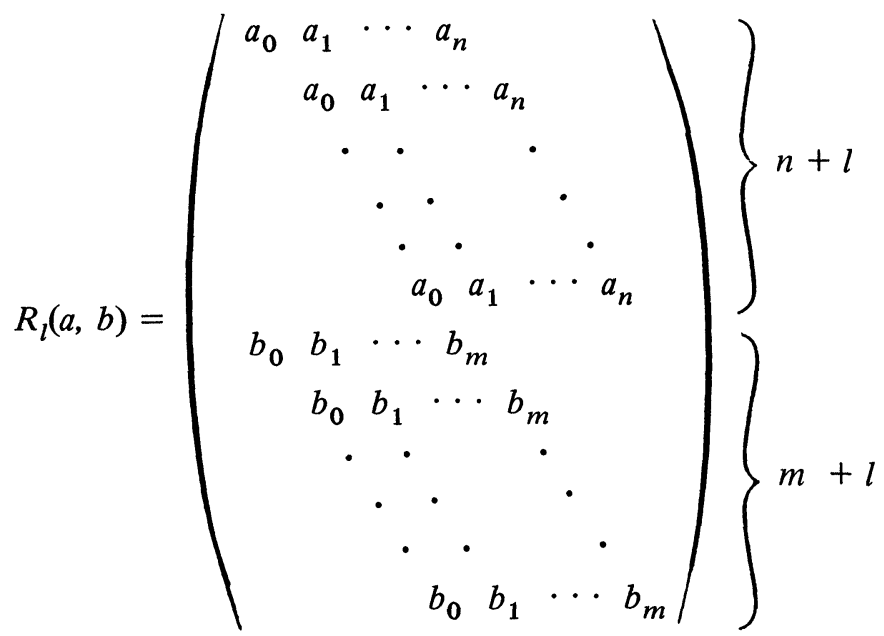

The second step consists of proving that the $\operatorname{dim} \operatorname{Ker} R_{l}(a \otimes I, I \otimes b)$ does not depend on $l$ and therefore

$$
\operatorname{dim} \operatorname{Ker} R_{l}(a \otimes I, I \otimes b)=\operatorname{dim} \operatorname{Ker} R(a \otimes I, I \otimes b) .
$$

Let us mention that the main theorem is connected with the theory of the equation $a(\lambda) x(\lambda)+y(\lambda) b(\lambda)=f(\lambda)$, where $f(\lambda)$ is the given and $x(\lambda), y(\lambda)$ are the unknown matrix polynomials.

All the detailed proofs will appear elsewhere together with some analogous results for the continuous case.

\section{REFERENCES}

1. I. C. Gohberg and G. Heining, Resultant matrix and its generalization. I: Resultant operator of matrix polynomials, Acta Sci. Math. (Szeged) 37 (1975), Fasc. 1-2, pp. 41-61. (Russian)

DEPARTMENT OF MATHEMATICAL SCIENCES, TEL-AVIV UNIVERSITY, RAMAT-AVIV, ISRAEL

DEPARTMENT OF MATHEMATICS, WEIZMANN INSTITUTE OF SCIENCE, REHOVOT, ISRAEL

DEPARTMENT OF MATHEMATICS, TECHNION, HAIFA, ISRAEL 